University of Nebraska - Lincoln

DigitalCommons@University of Nebraska - Lincoln

Faculty Publications, Department of Physics and Astronomy

Research Papers in Physics and Astronomy

$10-1-2004$

\title{
PHOTOMETRY OF TYPE II CEPHEIDS. I. THE LONG-PERIOD STARS
}

\author{
Edward G. Schmidt \\ University of Nebraska-Lincoln, eschmidt1@unl.edu \\ Dale Johnston \\ University of Nebraska-Lincoln, dalej96@gmail.com \\ Shawn Langan \\ University of Nebraska-Lincoln, nuaur@hotmail.com \\ Kevin M. Lee \\ University of Nebraska-Lincoln, klee6@unl.edu
}

Follow this and additional works at: https://digitalcommons.unl.edu/physicsfacpub

Part of the Physics Commons

Schmidt, Edward G.; Johnston, Dale; Langan, Shawn; and Lee, Kevin M., "PHOTOMETRY OF TYPE II CEPHEIDS. I. THE LONG-PERIOD STARS" (2004). Faculty Publications, Department of Physics and Astronomy. 26.

https://digitalcommons.unl.edu/physicsfacpub/26

This Article is brought to you for free and open access by the Research Papers in Physics and Astronomy at DigitalCommons@University of Nebraska - Lincoln. It has been accepted for inclusion in Faculty Publications, Department of Physics and Astronomy by an authorized administrator of DigitalCommons@University of Nebraska - Lincoln. 


\title{
PHOTOMETRY OF TYPE II CEPHEIDS. I. THE LONG-PERIOD STARS
}

\author{
Edward G. Schmidt, Dale Johnston, Shawn Langan, and Kevin M. Lee \\ Department of Physics and Astronomy, University of Nebraska, Lincoln, NE 68588-0111; eschmidt1@unl.edu, \\ dalej96@bigred.unl.edu, nuaur@hotmail.com,klee6@unl.edu \\ Received 2004 June 17; accepted 2004 July 8
}

\begin{abstract}
We present 1256 new photometric observations of 36 Cepheids with periods longer than 8 days. The majority are likely type II Cepheids, but we have included about a dozen classical Cepheids for comparison purposes, a few stars of uncertain type, and one putative RV Tauri star. We discuss the appearance of the light curves, the Fourier parameters, and the light-curve stability in terms of differentiation between type I and type II Cepheids. Although we encounter the same difficulties as previous investigators in using these parameters for this purpose, we are able to identify some stars of particular interest, including several likely type I Cepheids at large distances from the Galactic plane. Six stars with especially large period changes are identified and discussed.
\end{abstract}

Key words: Cepheids - stars: Population II

On-line material: machine-readable table

\section{INTRODUCTION}

Type II Cepheids (also referred to as Population II Cepheids or W Vir and BL Her stars, depending on their periods) differ from type I Cepheids (or classical Cepheids) in fundamental ways, including population type, metallicity, age, radius, luminosity, and mass. When these stars are members of star clusters, the distinction is clear; those in globular clusters are clearly type II Cepheids. However, distinguishing between type I and type II Cepheids in the field is generally difficult (see Harris 1985a, for example, for a discussion). Since some of the basic properties of globular cluster Cepheids are different from those of presumed type II Cepheids in the field (e.g., metallicity; Harris 1985a), this question needs to be addressed.

The reliable identification of field type II Cepheids is important for several reasons. Achieving a firm understanding of the population to which they belong is hampered by our inability to reliably identify individual stars in the field. This limits our understanding of their origin and evolution. In turn, any potential application of these stars as tracers of older populations and possibly the structure of the halo is compromised. It has been suggested that type II Cepheid pulsation is influenced by the onset of chaos as we go from short to longer period stars (Kovacs \& Buchler 1988). Again, observational tests of this interesting hypothesis depend strongly on the reliable identification of type II Cepheids.

Various observable parameters which have been proposed to distinguish between type I and type II Cepheids include the form of the light curve (based on the visual inspection, Fourier fits, or other light-curve parameters), the stability of the pulsation (as revealed by light-curve scatter or phase jitter), spectral features (emission lines, line doubling, differential motions), metallicity, space motions, and location.

Unfortunately, each of the first three of these properties is only useful in restricted period ranges. The last three properties, metallicity, space motion, and location, are compromised by the heterogeneity of the type II Cepheids. Their metallicities range from low to greater than solar, some have low space velocities (Harris \& Wallerstein 1984), and we would expect some to be found near the Galactic plane.

We have undertaken a project to obtain data, including new photometry, for a significant number of Cepheids with the aim of finding reliable ways to distinguish type I from type II stars. Although there is a large body of photometry of Cepheids in the literature, most of it is for type I Cepheids, and there are many likely type II Cepheids that lack significant modern photometry. In addition, combining data from various sources makes it difficult to separate intrinsic scatter in the light curve of a star from inhomogeneities in the observations. Finally, we are interested in the longer term stability of the pulsation, and new observations can be combined with existing data to study that question.

The present paper presents our new photometric data for Cepheids with periods longer than 8 days. Most are likely type II Cepheids based on their classification in the General Catalogue of Variable Stars (Kholopov 1985, 1987; hereafter GCVS) or their inclusion in Harris's (1985b) catalog. However, we have also included some classical Cepheids for comparison purposes, as well as some Cepheids of uncertain type. One RV Tau star was included since its period fell within the range of the type II Cepheids and the distinction between RV Tau stars and type II Cepheids is somewhat ambiguous.

\section{THE OBSERVATIONS}

The observations were made at Behlen Observatory with the $0.76 \mathrm{~m}$ telescope. Three different CCDs were used in the observations presented here: prior to 1999 May (JD 2,451,300) a Photometrics TI4849 CCD system (described by Schmidt 1991 and references therein); between 2000 January (JD $2,450,480$ ) and 2002 March (JD 2,452,344) a Santa Barbara Instruments ST-7 CCD; after 2002 April (JD 2,452,380) a Princeton Instruments VersArray:1300B CCD. The same methods were used for the observations and reductions as in previous papers (see Schmidt 1991 and references therein) except that after 1999 November IRAF ${ }^{1}$ was used for the reduction of the images and the extraction of instrumental magnitudes.

The stars discussed in this paper are listed in Table 1, where column (1) lists the names of the stars, column (2) lists their approximate periods, and column (3) gives the classifications

\footnotetext{
${ }^{1}$ IRAF is distributed by the National Optical Astronomy Observatory, which is operated by the Association of Universities for Research in Astronomy, Inc., under cooperative agreement with the National Science Foundation (NSF).
} 
TABLE 1

The Program Stars

\begin{tabular}{|c|c|c|c|c|c|c|c|c|c|c|}
\hline $\begin{array}{l}\text { Star } \\
(1)\end{array}$ & $\begin{array}{c}\text { Period } \\
\text { (days) } \\
(2)\end{array}$ & $\begin{array}{c}\text { GCVS Class } \\
\text { (3) }\end{array}$ & $\begin{array}{c}\text { Harris Class } \\
\text { (4) }\end{array}$ & $\begin{array}{l}n_{c} \\
(5)\end{array}$ & $\begin{array}{c}\sigma_{V} \\
(\mathrm{mmg}) \\
(6)\end{array}$ & $\begin{array}{c}\sigma_{R} \\
(\mathrm{mmg}) \\
(7)\end{array}$ & $\begin{array}{l}n_{n} \\
(8)\end{array}$ & $\begin{array}{c}\text { HJD }-2,400,000 \\
(9)\end{array}$ & $\begin{array}{c}N \\
(10)\end{array}$ & $\begin{array}{c}\text { Source of Phot. } \\
\text { (11) }\end{array}$ \\
\hline PZ Aql........... & 8.8 & CWA & $\ldots$ & 1 & 25 & 24 & 4 & $52,485-52,843$ & $54(12)$ & 1,2 \\
\hline \multirow[t]{2}{*}{ IX Cas ${ }^{\mathrm{a}} \ldots \ldots \ldots . . .}$. & 9.1 & CWA & II & 2 & 9 & 8 & 7 & $48,605-49,880$ & $69(10)$ & $1,3,4$ \\
\hline & & & & 3 & 8 & 14 & 4 & $52,194-52,914$ & (33) & \\
\hline CN Cep .......... & 9.5 & DCEP & $\ldots$ & 3 & 8 & 10 & 13 & $52,249-52,885$ & $102(30)$ & $1,5,6$ \\
\hline \multirow[t]{2}{*}{ AN Aur .......... } & 10.3 & DCEP & $\ldots$ & 2 & 4 & 15 & 3 & $48,647-50,803$ & $169(14)$ & $1,3,4,5,7$ \\
\hline & & & & 4 & 9 & 9 & $5^{\mathrm{b}}$ & $52,249-52,936$ & (34) & \\
\hline AL Vir ............ & 10.3 & CWA & II & 2 & 2 & 6 & $2^{\mathrm{b}}$ & $52,038-52,811$ & $76(22)$ & $1,3,8$ \\
\hline AP Her ........... & 10.4 & CWA & II & 2 & 6 & 8 & 14 & $50,501-52,865$ & $97(37)$ & $1,3,4$ \\
\hline BH Oph......... & 11.1 & CWA & II & 4 & 10 & 10 & 10 & $50,348-52,898$ & $54(38)$ & $1,9,10$ \\
\hline \multirow[t]{2}{*}{ QQ Per........... } & 11.2 & CEP & $\ldots$ & 2 & 14 & 9 & 4 & $47,872-51,921$ & $75(26)$ & $1,5,11$ \\
\hline & & & & 5 & 10 & 10 & 5 & $52,194-52,942$ & (29) & \\
\hline \multirow[t]{2}{*}{ AD Cam......... } & 11.3 & DCEP & $\ldots$ & 4 & $\ldots$ & $\ldots$ & $\mathrm{c}$ & $48,527-51,793$ & $149(13)$ & $1,3,5$ \\
\hline & & & & 3 & 11 & 11 & 6 & $52,249-52,942$ & (33) & \\
\hline EZ Cyg........... & 11.7 & DCEP & $\ldots$ & 3 & 12 & 12 & 3 & $51,047-52,898$ & $85(29)$ & 1,5 \\
\hline V775 Oph ..... & 12.2 & CWA & II & 2 & 14 & 14 & 9 & $51,013-52,811$ & $85(43)$ & 1,5 \\
\hline AS Vul ........... & 12.2 & DCEP & $\ldots$ & 3 & 18 & 17 & 5 & $50,768-52,885$ & 141(31) & 1,5 \\
\hline AL Lyr ........... & 13.0 & CWA & $\ldots$ & 1 & 8 & 11 & 12 & $49,880-52,913$ & $33(33)$ & 1 \\
\hline V916 Aq1 ...... & 13.4 & DCEP & $\ldots$ & 1 & 13 & 13 & $6^{\mathrm{b}}$ & $49,880-52,930$ & $152(28)$ & 1,5 \\
\hline V2338 Oph ... & 13.7 & CWA: & $\ldots$ & 5 & 12 & 10 & 6 & $52,415-52,914$ & $34(34)$ & 1 \\
\hline V801 Aql ...... & 14.2 & CWA & $\ldots$ & 4 & 13 & 12 & 5 & $52,485-52,930$ & $72(24)$ & 1,5 \\
\hline \multirow[t]{2}{*}{ CS Cas ............ } & 14.7 & CWA & II & 3 & 12 & 13 & 7 & $48,654-52,153$ & $57(18)$ & 1,3 \\
\hline & & & & 5 & 9 & 9 & 9 & $52,173-52,898$ & (29) & \\
\hline EU Cyg ......... & 15.0 & CEP & II & 2 & 12 & 14 & 4 & $52,194-52,898$ & $73(28)$ & 1,5 \\
\hline \multirow[t]{2}{*}{$\mathrm{CH}$ Cas........... } & 15.1 & DCEP & $\ldots$ & 2 & 16 & 12 & 7 & $48,642-51,075$ & 131(12) & $1,3,5,10$ \\
\hline & & & & 4 & 15 & 20 & 4 & $52,194-52,905$ & (32) & \\
\hline V845 Her ....... & 15.5 & CWA & $\ldots$ & 3 & 18 & 29 & 4 & $51,793-52,934$ & $29(29)$ & 1 \\
\hline AL Sct............. & 15.6 & CWA & II & 2 & 15 & 6 & 2 & $52,851-52,913$ & $18(18)$ & 1 \\
\hline SZ Mon .......... & 16.3 & RVA & $\ldots$ & 4 & 13 & 13 & 5 & $52,249-52,933$ & $67(27)$ & 1,8 \\
\hline V478 Oph ..... & 16.3 & CWA & II & 2 & 10 & 13 & 8 & $51,793-52,811$ & $37(25)$ & $1,3,9$ \\
\hline \multirow[t]{2}{*}{ СР Сер............ } & 17.9 & DCEP & $\ldots$ & 3 & 5 & 11 & 5 & $48,654-51,800$ & $117(13)$ & 1,5 \\
\hline & & & & 4 & 17 & 13 & 4 & $52,218-52,885$ & (25) & \\
\hline DR Cep .......... & 19.1 & DCEP & II & 2 & 13 & 11 & $6^{\mathrm{b}}$ & $51,914-52,898$ & $131(30)$ & 1,5 \\
\hline \multirow[t]{2}{*}{ KX Cyg.......... } & 20.1 & DCEP & $\ldots$ & 2 & 22 & 28 & 3 & $48,574-50,606$ & 142(8) & 1,5 \\
\hline & & & & 3 & 28 & 28 & $3^{b}$ & $52,218-52,930$ & (23) & \\
\hline MZ Cyg......... & 21.4 & CWA: & II & 4 & 12 & 10 & 14 & $52,038-52,898$ & $103(40)$ & $1,3,5$ \\
\hline PP Aql ............ & 24.0 & DCEP & II & 5 & 23 & 26 & 3 & $52,082-52,858$ & $52(24)$ & $1,2,3,5$ \\
\hline CC Lyr ........... & 24.2 & CWA & II & 2 & 11 & 11 & 12 & $50,292-52,865$ & $56(47)$ & 1,3 \\
\hline \multirow[t]{2}{*}{ OT Per............ } & 26.1 & DCEP & $\ldots$ & 2 & 14 & 13 & 5 & $48,606-48,720$ & $100(11)$ & 1,5 \\
\hline & & & & 3 & 13 & 13 & 3 & $52,249-52,942$ & (36) & \\
\hline TW Cap.......... & 28.6 & CWA & II & 4 & 15 & 14 & 8 & $52,055-52,942$ & $66(49)$ & 1,3 \\
\hline NN Vul.......... & 30.8 & CWA & $\ldots$ & 2 & 12 & 13 & 12 & $51,815-52,905$ & $70(52)$ & 1,5 \\
\hline V609 Cyg...... & 31.1 & DCEP & $\ldots$ & 4 & 22 & 19 & 3 & $50,768-52,905$ & $164(33)$ & $1,5,10$ \\
\hline IU Cyg .......... & 31.4 & CW: & II & 2 & 14 & 13 & 9 & $51,793-52,908$ & $43(22)$ & 1,5 \\
\hline EV Aql .......... & 38.8 & CEP & II & 2 & 12 & 16 & 5 & $49,880-52,865$ & $305(29)$ & $1,2,3,5,9,10$ \\
\hline ET Vul........... & 53.5 & CWA & II & 3 & 12 & 11 & 17 & $50,319-52,905$ & 93(43) & $1,3,5,10$ \\
\hline
\end{tabular}

a IX Cas is a member of a single-line spectroscopic binary (Harris \& Welch 1989).

b The $V$ magnitudes from the present photometry differed systematically from the earlier photometry. Accordingly, their zero point was adjusted to produce agreement with the earlier photometry.

c No photometric nights are available. The zero point is set to make mean of variable equal that from later photometry.

REFERENCES.-(1) New Behlen Observatory photometry; (2) Pel 1976 (Walraven photometry transformed to the Johnson system using $V_{J}=6.874-$

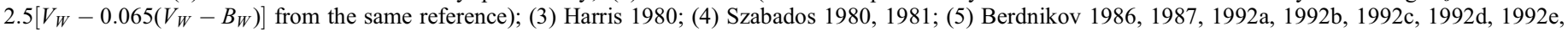

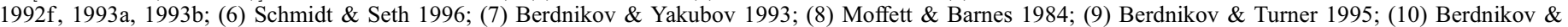
Voziakova 1995; (11) Schmidt et al. 1995.

of the stars from the GCVS. A "II" in column (4) identifies stars that appear in Table I of Harris's (1985b) catalog of type II Cepheids. He selected these objects on the basis of distance from the Galactic plane. We note that SZ Mon would not have been considered by Harris because it is listed as an RV Tauri star (RVA) in the GCVS. However, using Harris's criteria, it would have been rejected because of its small distance from the Galactic plane.
Those type II Cepheids that occur near the Galactic plane are not included in Harris's catalog. There are also indications that short-period type I Cepheids are sometimes found well away from the Galactic plane (Balog et al. 1997; Fernie \& Ehlers 1999; Schmidt et al. 2003a). This is likely to be true among long-period stars as well. To minimize confusion and to avoid biasing our discussion of the types of individual stars, we refer to the stars from Harris's catalog (identified by "II" 
TABLE 2

PноTOMETRIC DAta

\begin{tabular}{ccccc}
\hline \hline Star & HJD & $V$ & $R$ & $V-R$ \\
\hline PZ Aq1.............. & $52,485.829$ & 11.760 & 10.917 & 0.842 \\
PZ Aq1............ & $52,488.784$ & 11.361 & 10.538 & 0.824 \\
PZ Aq1............. & $52,500.717$ & 11.942 & 10.986 & 0.956 \\
PZ Aq1.............. & $52,501.672$ & 12.102 & 11.155 & 0.946 \\
PZ Aq1............. & $52,535.615$ & 11.735 & 10.790 & 0.946 \\
\hline
\end{tabular}

Note.-Table 2 is presented in its entirety in the electronic edition of the Astronomical Journal. A portion is shown here for guidance regarding its form and content. in col. [4] of Table 1) as high- $Z$ Cepheids and those not in the catalog as low- $Z$ Cepheids.

Information about the comparison stars is provided in columns (5)-(8). Column (5) lists the number of comparison stars used for each field. Columns (6) and (7) contain the standard errors of the adopted $V$ and $R$ magnitudes of the comparison stars. Column (8) gives the number of photometric nights included in calculating the comparison star magnitudes. To exploit the larger fields of the ST-7 and VersArray:1300B CCDs, new comparison stars were selected for some fields. When the same variable was observed with two sets of comparison stars this is reflected by two lines in Table 1 .

Because Behlen Observatory is a low-quality photometric site, placing the comparison stars on the standard system

TABLE 3

The Light-Curve Parameters

\begin{tabular}{|c|c|c|c|c|c|c|c|c|c|c|}
\hline $\begin{array}{l}\text { Star } \\
(1)\end{array}$ & $\begin{array}{c}\text { Period } \\
\text { (days) } \\
(2)\end{array}$ & $\begin{array}{l}\sigma_{P} \\
(3)\end{array}$ & $\begin{array}{c}\mathrm{HJD}_{\max }-2,400,000 \\
\text { (4) }\end{array}$ & $\begin{array}{c}\text { Light-Curve Type }{ }^{\mathrm{a}} \\
\text { (5) }\end{array}$ & $\begin{array}{c}\langle V\rangle \\
(\mathrm{mag}) \\
(6)\end{array}$ & $\begin{array}{c}\langle R\rangle \\
(\mathrm{mag}) \\
(7)\end{array}$ & $\begin{array}{c}\Delta V \\
(\mathrm{mag}) \\
(8)\end{array}$ & $\begin{array}{c}\Delta R \\
(\mathrm{mag}) \\
(9)\end{array}$ & $\begin{array}{l}\phi_{\min } \\
(10)\end{array}$ & $\begin{array}{l}\text { Notes } \\
(11)\end{array}$ \\
\hline 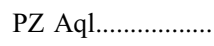 & 8.7543 & 1 & 52811.5 & $\mathrm{D}$ & 11.66 & 10.78 & 0.78 & 0.62 & 0.66 & $\mathrm{~b}$ \\
\hline 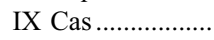 & 9.1480 & 15 & 52820.9 & A & 11.48 & 11.08 & 0.57 & 0.46 & 0.50 & $\mathrm{c}$ \\
\hline 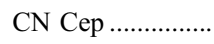 & 9.5041 & 3 & 52587.9 & A & 12.36 & 11.22 & 0.61 & 0.50 & 0.72 & $\mathrm{~b}$ \\
\hline 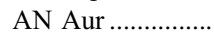 & 10.2891 & 3 & 52709.5 & $\mathrm{C}$ & 10.46 & 9.69 & 0.71 & 0.56 & 0.53 & $\mathrm{~b}, \mathrm{~d}$ \\
\hline AL Vir ........................ & 10.3053 & 2 & 52785.1 & $\mathrm{D}$ & 9.53 & 9.13 & 0.82 & 0.64 & 0.64 & b \\
\hline AP Her ..................... & 10.400 & 5 & 52502.5 & $\mathrm{D}$ & 10.81 & 10.29 & 0.92 & 0.67 & 0.73 & $\mathrm{c}$ \\
\hline 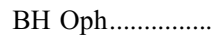 & 11.059 & 1 & 52872.6 & $\mathrm{D}$ & 12.06 & 11.65 & 0.91 & 0.74 & 0.77 & $\mathrm{~b}, \mathrm{~d}$ \\
\hline QQ Per ...................... & 11.189 & 2 & 52683.7 & $\mathrm{~A}$ & 13.71 & 13.15 & 0.61 & 0.55 & 0.74 & $\mathrm{c}$ \\
\hline 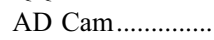 & 11.2619 & 2 & 52616.6 & $\mathrm{Db}$ & 12.53 & 11.55 & 1.13 & 0.85 & 0.69 & $\mathrm{~b}$ \\
\hline 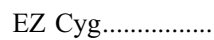 & 11.6608 & 8 & 52817.9 & $\mathrm{Db}$ & 11.04 & 10.19 & 0.96 & 0.74 & 0.63 & $\mathrm{~b}$ \\
\hline 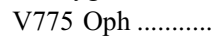 & 12.18 & 1 & 52772.2 & A & 13.42 & 12.89 & 0.51 & 0.43 & 0.42 & $\mathrm{c}$ \\
\hline 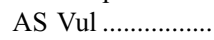 & 12.2252 & 4 & 52884.9 & $\mathrm{Db}$ & 12.27 & 11.14 & 0.93 & 0.76 & 0.66 & $\mathrm{~b}$ \\
\hline 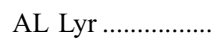 & 12.978 & 2 & 52912.6 & $\mathrm{~A} / \mathrm{B}$ & 12.02 & 11.36 & 0.81 & 0.70 & 0.56 & $\mathrm{~b}$ \\
\hline 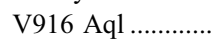 & 13.4430 & 4 & 52822.1 & $\mathrm{Db}$ & 10.78 & 9.80 & 1.02 & 0.78 & 0.67 & $\mathrm{~b}$ \\
\hline V2338 Oph .............. & 13.665 & 10 & 52805.9 & $\mathrm{~A}$ & 11.84 & 11.24 & 0.74 & 0.63 & 0.48 & $\mathrm{~b}$ \\
\hline 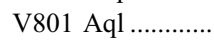 & 14.160 & 2 & 52856.9 & $\mathrm{~A} / \mathrm{B}$ & 13.45 & 12.74 & 0.70 & 0.58 & 0.52 & e \\
\hline 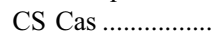 & 14.7333 & 4 & 52639.6 & B & 12.07 & 11.58 & 1.36 & 1.11 & 0.64 & $\mathrm{~b}$ \\
\hline EU Cyg .................... & 14.987 & 1 & 52755.8 & $\mathrm{Db}$ & 13.86 & 12.87 & 1.00 & 0.80 & 0.72 & $\mathrm{~b}$ \\
\hline 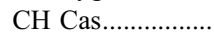 & 15.0900 & 5 & 52664.8 & $\mathrm{Db}:$ & 10.99 & 9.95 & 1.10 & 0.83 & 0.62 & $\mathrm{~b}$ \\
\hline 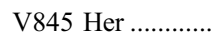 & 15.50 & 3 & 52753.3 & $\mathrm{~A}$ & 13.59 & 13.11 & 0.91 & 0.80 & 0.46 & $\mathrm{~b}$ \\
\hline 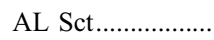 & 15.5751 & $\ldots$ & 52904.3 & $\ldots$ & 13.85 & 13.14 & 0.92 & 0.80 & 0.62 & $\mathrm{f}$ \\
\hline 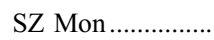 & 16.334 & 3 & 52710.6 & $\mathrm{~A}$ & 10.06 & 9.53 & 1.28 & 1.27 & 0.56 & $\mathrm{c}, \mathrm{d}$ \\
\hline 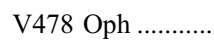 & 16.347 & 1 & 52810.1 & A & 12.92 & 12.25 & 1.00 & 0.87 & 0.51 & $\mathrm{~b}$ \\
\hline 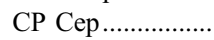 & 17.8645 & 10 & 52521.9 & $\mathrm{Db}$ & 10.56 & 9.60 & 0.82 & 0.63 & 0.62 & $\mathrm{~b}$ \\
\hline 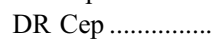 & 19.0775 & 15 & 52851.4 & $\mathrm{Db}$ & 12.85 & 12.02 & 1.09 & 0.84 & 0.75 & $\mathrm{~b}$ \\
\hline 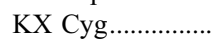 & 20.052 & 1 & 52250.8 & $\mathrm{Db}$ & 11.95 & 10.51 & 1.14 & 0.83 & 0.73 & $\mathrm{~b}$ \\
\hline MZ Cyg.................. & 21.41 & 5 & 52580.2 & A & 11.60 & 11.08 & 1.56 & 1.33 & 0.78 & $\mathrm{c}, \mathrm{d}$ \\
\hline PP Aql & 24.050 & 4 & 52534.3 & A & 11.79 & 11.12 & 1.53 & 0.98 & 0.63 & $\mathrm{~b}, \mathrm{~d}$ \\
\hline 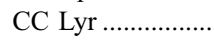 & 24.226 & 6 & 52038.7 & B & 11.98 & 11.63 & 0.63 & 0.57 & 0.76 & $b, d$ \\
\hline 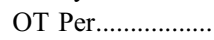 & 26.094 & 2 & 52712.9 & $\mathrm{D}$ & 13.50 & 12.12 & 1.02 & 0.81 & 0.87 & $\mathrm{~b}$ \\
\hline 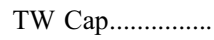 & 28.56 & 1 & 52810.8 & B & 10.49 & 10.11 & 1.36 & 1.06 & 0.74 & $\mathrm{~d}, \mathrm{~g}$ \\
\hline 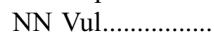 & 30.819 & 6 & 52904.7 & B & 14.33 & 13.40 & 1.05 & 0.97 & 0.78 & $b, d$ \\
\hline 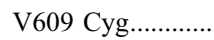 & 31.087 & 4 & 52859.8 & $\mathrm{D}$ & 11.07 & 9.81 & 1.26 & 0.85 & 0.83 & $\mathrm{~b}$ \\
\hline 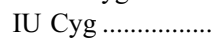 & 31.354 & 1 & 52579.6 & B & 13.26 & 12.60 & 1.02 & 0.83 & 0.75 & $\mathrm{~b}$ \\
\hline 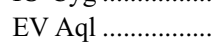 & 38.767 & $\ldots$ & 52500.8 & $\mathrm{D}$ & 11.92 & 11.02 & 0.77 & 0.57 & 0.78 & $\mathrm{~d}, \mathrm{~h}$ \\
\hline 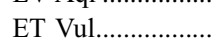 & 53.493 & $\cdots$ & 52810.0 & A & 12.18 & 11.21 & 0.51 & 0.39 & 0.63 & $\mathrm{~d}, \mathrm{~h}$ \\
\hline
\end{tabular}

a The light-curve types are defined in the text.

${ }^{b}$ Period and other light-curve parameters are based on all the data referenced in Table 1.

${ }^{c}$ No unique period could be found that fits all of the data satisfactorily. The value listed is only valid for the recent data and only that data are used in deriving the light-curve parameters and are plotted in Fig. 1.

d Alternation of cycles is possible. The period refers to the short cycle.

e The period and epoch are based on all of the data referenced in Table 1. However, the light-curve parameters are based only on the present photometry and only the present photometry is plotted in Fig. 1.

${ }^{\mathrm{f}}$ Too little data to determine a period. The GCVS value was adopted.

${ }^{g}$ Several periods were found that fitted the data equally well. The one closest to the GCVS period was adopted.

${ }^{\mathrm{h}}$ The GCVS period was used, and only the present data are plotted in Fig. 1 and used to derive the light-curve parameters. 

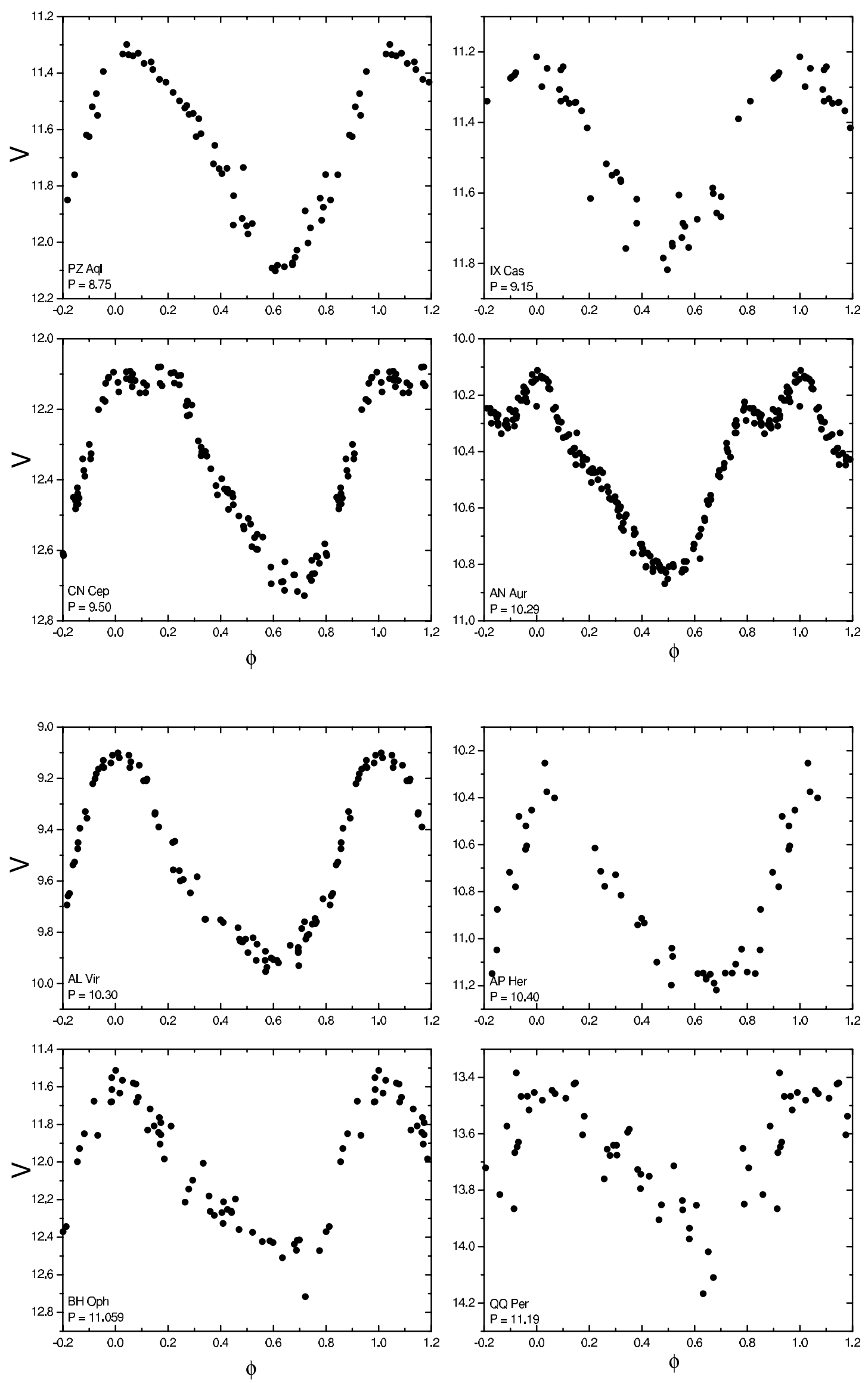

Fig. 1. $-V$ magnitude light curves for all of the stars listed in Table 1. 

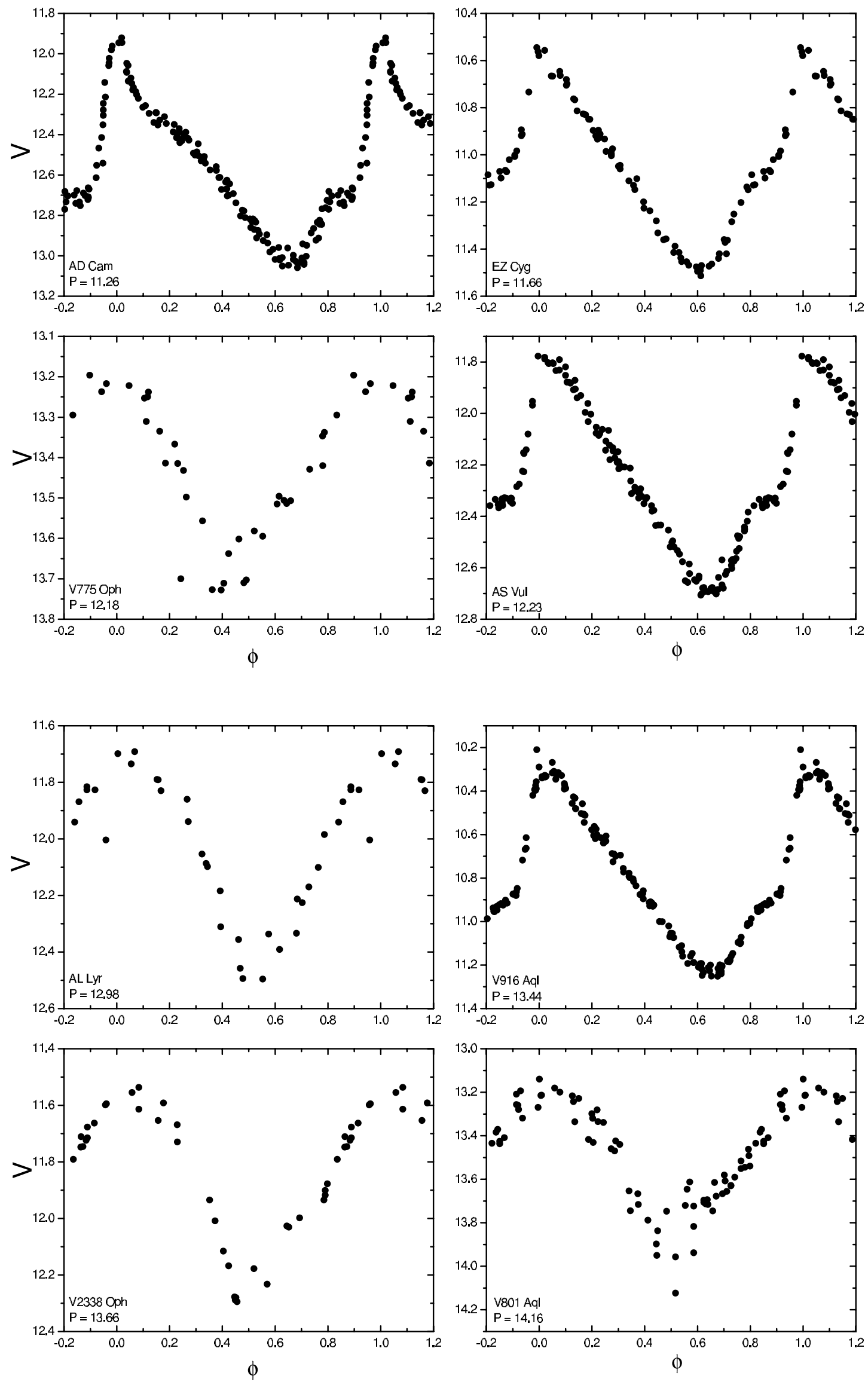

FIG. 1.-Continued 

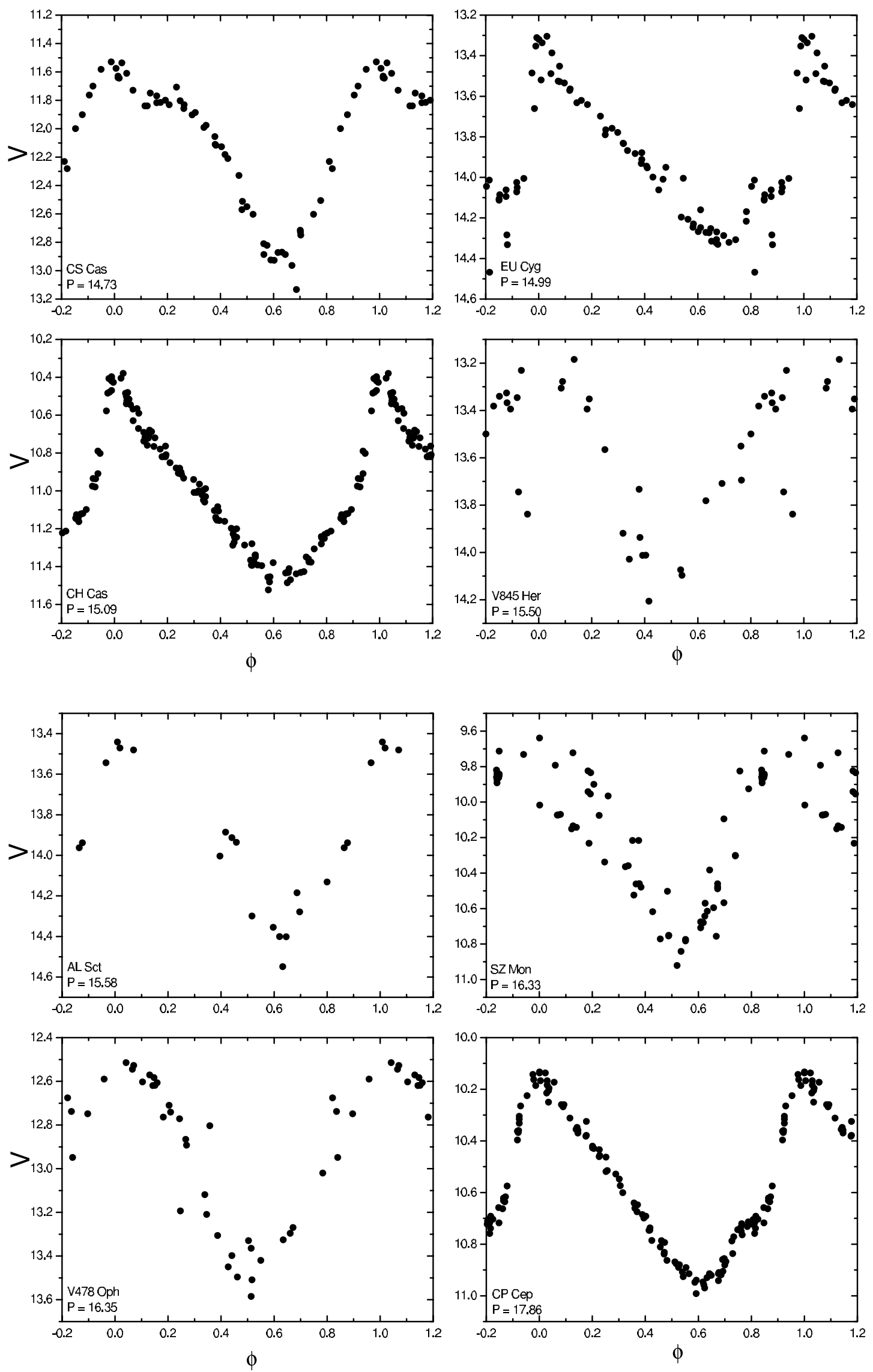

Fig. 1.—Continued 

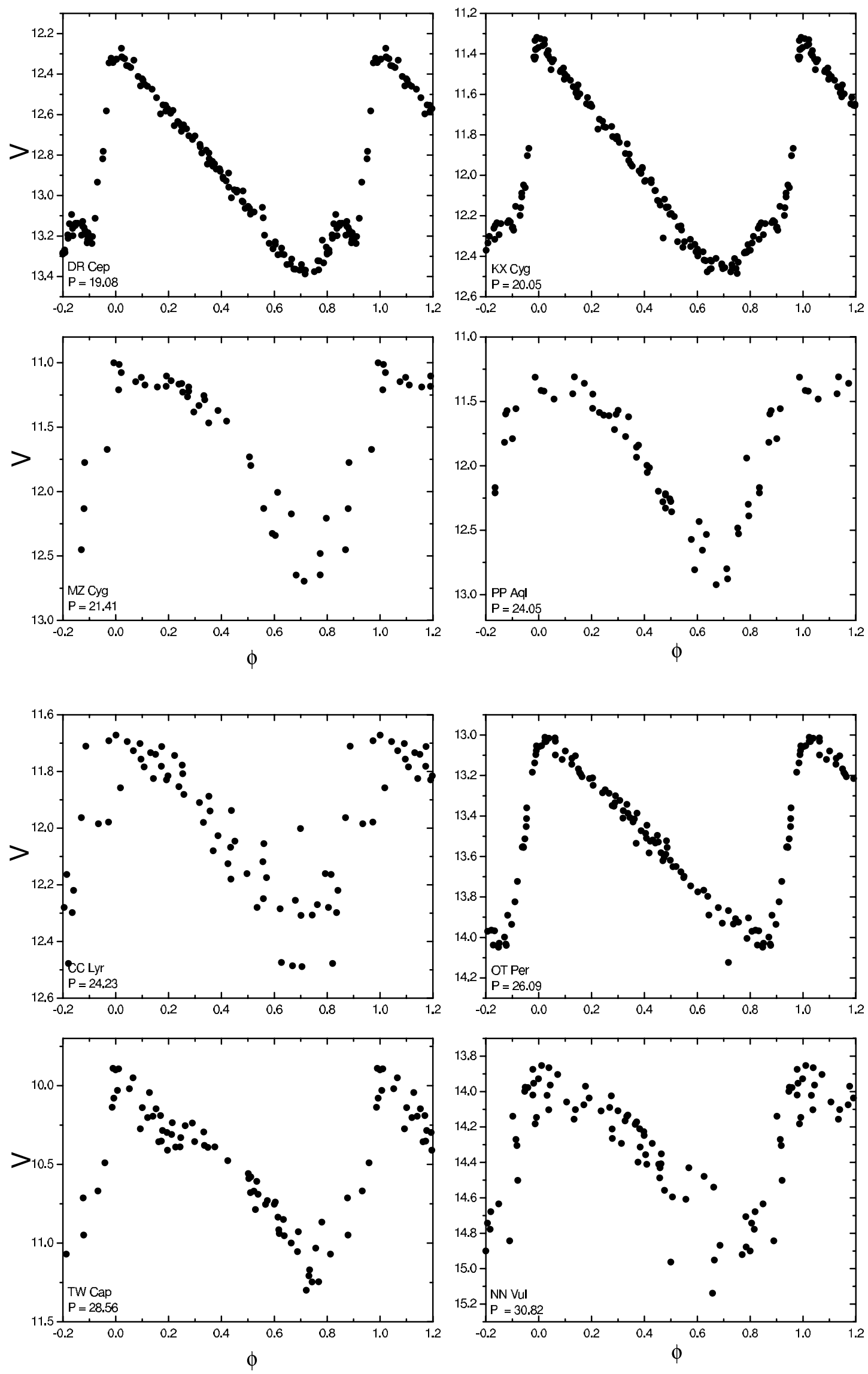

FIG. 1.—Continued 

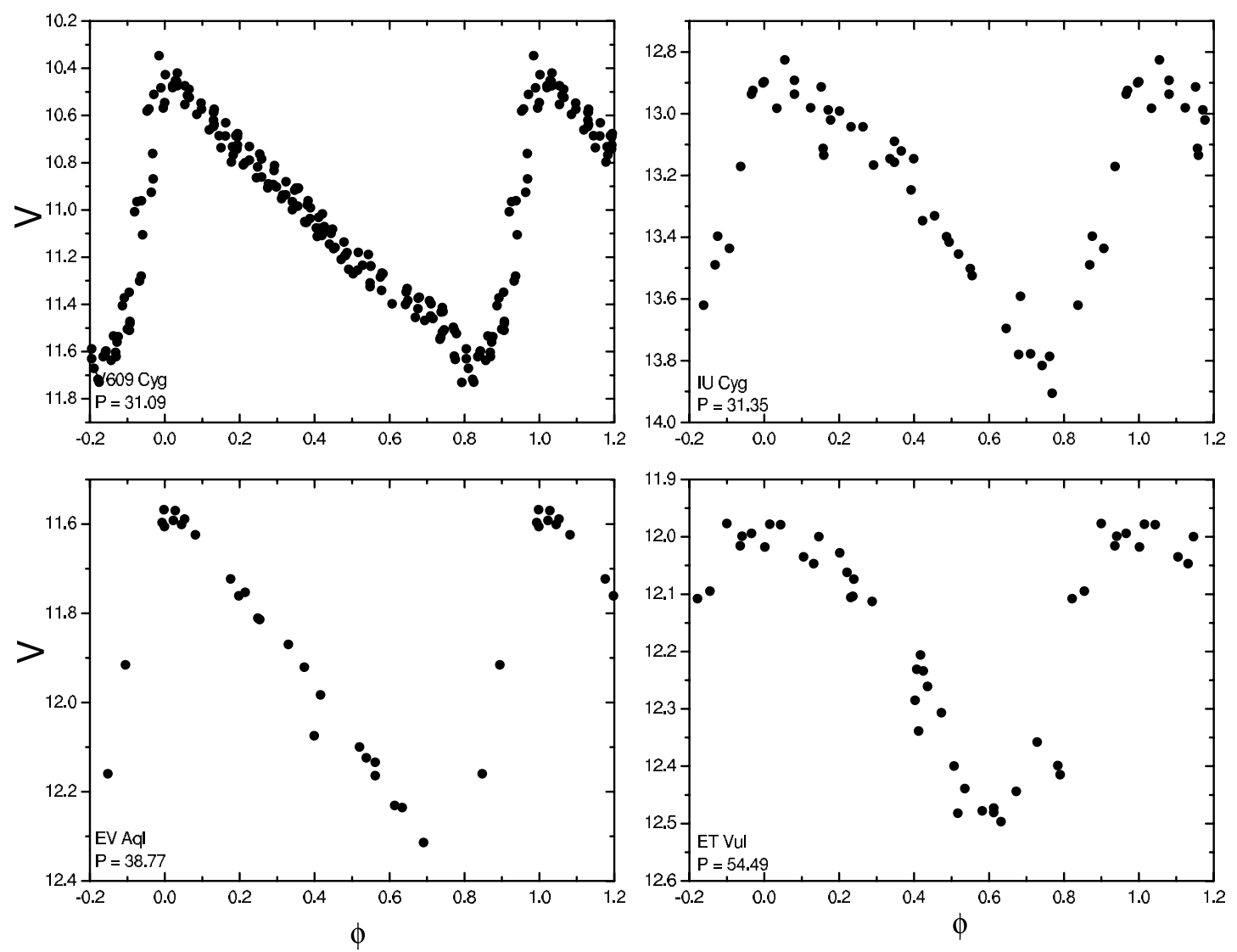

Fig. 1.-Continued

presents difficulties. As a check, we compared our $V$ light curves with those from earlier photometry (references in col. [11]) to identify any zero-point differences. For five stars, AN Aur since JD 2,452,249, and AL Vir, V916 Aql, DR Cep, and KX Cyg since JD 2,452,218, discrepancies were found that ranged up to $0.09 \mathrm{mag}$. In those cases, the zero point of our $V$ magnitudes was adjusted to produce agreement with the earlier photometry. Unfortunately, no such check is possible for the $R$ magnitudes. For AD Cam prior to JD 2,451,793, we had no data from a photometric night. In that case the $V$ magnitudes of the variable were compared with those from our later photometry, and the zero point was set to achieve agreement.

In column (9) we list the range of Julian Dates of our observations. In our analysis we have incorporated $V$ photometry from the literature for most of the stars, in addition to our new observations. In column (10) the first number indicates the total number of light-curve points used, while the number in parentheses is the number of new points presented here. Finally, column (11) provides references to the sources of the photometry.

In Table 2 we list the new observations.

\section{PROPERTIES OF THE LIGHT CURVES}

In Table 3 we list a variety of parameters determined from the photometry. Column (1) contains the name of the star, while column (2) gives a newly determined period. A first estimate of the period was found using the data-compensated discrete Fourier transform method described by Ferraz-Mello (1981). This was then adjusted by a small amount to produce a minimum of scatter between the newest and oldest observations.
The uncertainty was found by applying small changes in period until scatter in the light curve was unacceptable. Thus, the errors of the periods (col. [3] in units of the last cited decimal place in col. [2]) can be considered maximum errors rather than more formal standard deviations. As indicated by the notes to the table, it was not possible to derive a unique period for some stars, so only recent data was used.

There are a few stars that show evidence of alternation of cycles, reminiscent of RV Tau stars. In those cases, identified by a footnote, we have listed the period between successive minima (also referred to as the "short period" and equal to half of the "formal period" in the terminology of the GCVS) even though it produces more scatter in the light curves.

In column (4) we list the epoch of maximum based on the most recent photometry. There is always uncertainty in determining the time of maximum light in the presence of scatter and limited phase coverage. An additional source of ambiguity is the presence of broad, flat, or even double maxima in some stars. The reader can see how these issues were handled by examining the plotted light curves.

Although classing light curves according to appearance is subjective and does not yield quantitative parameters for further analysis, it can provide initial insights into relationships among various stars (for example, see Diethelm 1990 and Schmidt et al. 2003b). Based on the discussions of PayneGaposchkin (1956) and Kwee (1967) and an examination of our light curves, we have defined the following light-curve types:

A: Stars with a broad relatively flat maximum and a symmetric minimum that is usually rather narrow and often located near phase 0.5 . This category is similar to Payne-Gaposchkin's "broad maximum" group and Kwee's "flat-topped variables." 
TABLE 4

The Fourier Parameters

\begin{tabular}{|c|c|c|c|c|c|c|c|c|c|c|c|c|c|}
\hline $\begin{array}{c}\text { Star } \\
(1)\end{array}$ & $\begin{array}{l}R_{21} \\
(2)\end{array}$ & $\begin{array}{c}\sigma \\
(3)\end{array}$ & $\begin{array}{l}R_{31} \\
(4)\end{array}$ & $\begin{array}{c}\sigma \\
(5)\end{array}$ & $\begin{array}{l}R_{41} \\
(6)\end{array}$ & $\begin{array}{c}\sigma \\
(7)\end{array}$ & $\begin{array}{l}\phi_{21} \\
(8)\end{array}$ & $\begin{array}{c}\sigma \\
(9)\end{array}$ & $\begin{array}{c}\phi_{31} \\
(10)\end{array}$ & $\begin{array}{c}\sigma \\
(11)\end{array}$ & $\begin{array}{c}\phi_{41} \\
(12)\end{array}$ & $\begin{array}{c}\sigma \\
(13)\end{array}$ & $\begin{array}{c}\sigma_{V} \\
(14)\end{array}$ \\
\hline PZ Aql........................ & 0.13 & 0.02 & 0.05 & 0.02 & 0.06 & 0.02 & 5.07 & 0.18 & 1.26 & 0.46 & 6.04 & 0.40 & 0.039 \\
\hline IX Cas ........................... & 0.08 & 0.03 & 0.02 & 0.03 & 0.10 & 0.03 & 5.30 & 0.35 & 2.37 & 1.37 & 0.93 & 0.31 & 0.034 \\
\hline 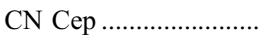 & 0.14 & 0.01 & 0.10 & 0.01 & 0.08 & 0.01 & 4.95 & 0.08 & 3.97 & 0.12 & 0.87 & 0.15 & 0.024 \\
\hline 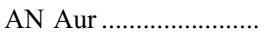 & 0.13 & 0.01 & 0.14 & 0.01 & 0.11 & 0.01 & 5.55 & 0.07 & 4.86 & 0.07 & 2.00 & 0.09 & 0.033 \\
\hline AL Vir ........................... & 0.27 & 0.02 & 0.04 & 0.02 & 0.04 & 0.02 & 3.78 & 0.06 & 1.11 & 0.36 & 5.89 & 0.40 & 0.036 \\
\hline AP Her ............................ & 0.26 & 0.04 & 0.13 & 0.04 & 0.02 & 0.04 & 4.03 & 0.19 & 1.78 & 0.35 & 5.05 & 1.78 & 0.062 \\
\hline 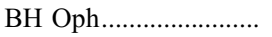 & 0.35 & 0.04 & 0.08 & 0.04 & 0.07 & 0.04 & 4.06 & 0.12 & 2.14 & 0.45 & 1.10 & 0.51 & 0.076 \\
\hline QQ Per ........................ & 0.26 & 0.08 & 0.09 & 0.08 & $\ldots$ & $\ldots$ & 4.97 & 0.34 & 6.12 & 0.89 & $\ldots$ & $\ldots$ & 0.096 \\
\hline 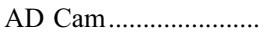 & 0.25 & 0.01 & 0.15 & 0.01 & 0.18 & 0.01 & 4.66 & 0.05 & 1.69 & 0.08 & 5.66 & 0.07 & 0.029 \\
\hline EZ Cyg........................... & 0.12 & 0.01 & 0.12 & 0.01 & 0.11 & 0.01 & 4.26 & 0.08 & 0.52 & 0.08 & 4.62 & 0.09 & 0.024 \\
\hline V775 Oph ...................... & 0.14 & 0.07 & 0.09 & 0.06 & 0.05 & 0.06 & 1.49 & 0.47 & 1.44 & 0.75 & 5.97 & 1.33 & 0.062 \\
\hline AS Vul .......................... & 0.16 & 0.01 & 0.14 & 0.01 & 0.12 & 0.01 & 4.30 & 0.06 & 0.73 & 0.07 & 5.17 & 0.09 & 0.019 \\
\hline AL Lyr ........................ & 0.15 & 0.03 & 0.05 & 0.03 & 0.04 & 0.03 & 0.55 & 0.21 & 1.53 & 0.59 & 2.93 & 0.73 & 0.043 \\
\hline V916 Aq1 ..................... & 0.20 & 0.01 & 0.13 & 0.01 & 0.11 & 0.01 & 4.30 & 0.04 & 1.20 & 0.06 & 5.39 & 0.07 & 0.025 \\
\hline V2338 Oph ................ & 0.16 & 0.03 & 0.18 & 0.03 & 0.06 & 0.03 & 1.01 & 0.16 & 1.54 & 0.16 & 1.82 & 0.41 & 0.034 \\
\hline V801 Aq1 ..................... & 0.13 & 0.03 & 0.14 & 0.03 & 0.05 & 0.03 & 1.72 & 0.26 & 2.04 & 0.25 & 3.48 & 0.63 & 0.027 \\
\hline CS Cas ............................. & 0.33 & 0.02 & 0.05 & 0.02 & 0.06 & 0.02 & 5.67 & 0.05 & 2.80 & 0.28 & 0.44 & 0.24 & 0.050 \\
\hline EU Cyg ....................... & 0.28 & 0.04 & 0.16 & 0.03 & 0.19 & 0.03 & 4.47 & 0.14 & 1.73 & 0.22 & 5.94 & 0.22 & 0.052 \\
\hline CH Cas.......................... & 0.24 & 0.01 & 0.17 & 0.01 & 0.13 & 0.01 & 4.28 & 0.06 & 1.42 & 0.09 & 5.35 & 0.11 & 0.030 \\
\hline V845 Her ...................... & 0.22 & 0.06 & 0.06 & 0.06 & 0.14 & 0.07 & 0.65 & 0.32 & 2.37 & 1.09 & 5.76 & 0.52 & 0.091 \\
\hline AL Sct......................... & $\ldots$ & $\ldots$ & $\ldots$ & $\ldots$ & $\ldots$ & $\ldots$ & $\ldots$ & $\ldots$ & $\ldots$ & $\ldots$ & $\ldots$ & $\ldots$ & 0.101 \\
\hline SZ Mon ......................... & 0.34 & 0.05 & 0.06 & 0.05 & 0.06 & 0.05 & 5.42 & 0.18 & 1.80 & 0.86 & 4.44 & 0.82 & 0.156 \\
\hline V478 Oph ....................... & 0.09 & 0.04 & 0.03 & 0.04 & 0.05 & 0.04 & 0.93 & 0.40 & 2.56 & 1.13 & 2.86 & 0.69 & 0.067 \\
\hline 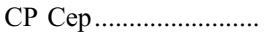 & 0.18 & 0.01 & 0.12 & 0.01 & 0.11 & 0.01 & 4.30 & 0.05 & 1.35 & 0.08 & 5.54 & 0.09 & 0.021 \\
\hline DR Cep ......................... & 0.32 & 0.01 & 0.14 & 0.01 & 0.14 & 0.01 & 4.62 & 0.04 & 2.35 & 0.08 & 0.16 & 0.09 & 0.040 \\
\hline KX Cyg............................ & 0.28 & 0.01 & 0.17 & 0.01 & 0.14 & 0.01 & 4.46 & 0.05 & 2.06 & 0.08 & 0.03 & 0.03 & 0.046 \\
\hline 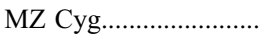 & 0.28 & 0.05 & 0.07 & 0.05 & 0.06 & 0.05 & 5.57 & 0.20 & 3.68 & 0.70 & 2.13 & 0.84 & 0.154 \\
\hline PP Aql ........................ & 0.23 & 0.04 & 0.08 & 0.04 & 0.05 & 0.04 & 5.51 & 0.17 & 4.39 & 0.47 & 3.77 & 0.72 & 0.071 \\
\hline 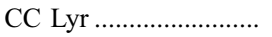 & 0.20 & 0.07 & 0.13 & 0.07 & 0.04 & 0.06 & 4.72 & 0.35 & 3.80 & 0.55 & 2.96 & 1.67 & 0.094 \\
\hline OT Per........................... & 0.41 & 0.01 & 0.24 & 0.01 & 0.16 & 0.01 & 4.71 & 0.04 & 2.99 & 0.06 & 1.37 & 0.09 & 0.027 \\
\hline TW Cap...................... & 0.45 & 0.04 & 0.18 & 0.04 & 0.12 & 0.04 & 5.25 & 0.11 & 3.20 & 0.23 & 0.55 & 0.34 & 0.091 \\
\hline NN Vul......................... & 0.34 & 0.06 & 0.22 & 0.06 & 0.02 & 0.05 & 5.10 & 0.19 & 3.69 & 0.29 & 2.71 & 2.98 & 0.147 \\
\hline 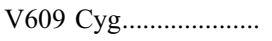 & 0.46 & 0.02 & 0.29 & 0.02 & 0.15 & 0.02 & 4.57 & 0.06 & 2.99 & 0.09 & 1.36 & 0.15 & 0.042 \\
\hline IU Cyg ........................ & 0.34 & 0.04 & 0.18 & 0.04 & 0.03 & 0.04 & 5.19 & 0.13 & 3.54 & 0.22 & 2.17 & 1.10 & 0.097 \\
\hline EV Aq1 ........................... & 0.39 & 0.01 & 0.16 & 0.01 & 0.05 & 0.01 & 4.86 & 0.04 & 3.32 & 0.08 & 1.97 & 0.26 & 0.014 \\
\hline ET Vul.......................... & 0.26 & 0.05 & 0.07 & 0.05 & $\ldots$ & $\ldots$ & 5.29 & 0.21 & 4.09 & 0.66 & $\ldots$ & $\ldots$ & 0.049 \\
\hline
\end{tabular}

The light curve of $\mathrm{CN}$ Cas in Figure 1 provides a good example.

B: Stars with a bump during declining light. They sometimes show the a symmetric minimum similar to the type A stars. CS Cas is a typical example of this type.

C: Stars with a relatively slow rise that is interrupted by a significant bump. The minimum is symmetric and located near the middle of the cycle. The only star with a light curve of this type in our sample is AN Aur.

D: Stars with a rapid rise to maximum and slow decline. These light curves are similar to those of the Bailey type $b$ RR Lyrae stars. There are a number of light curves in Figure 1 that clearly belong to this type, including that of OT Per. In some cases there is a significant bump after minimum, which is denoted by Db. DR Cep exhibits a good example of a light curve of this type.

The light-curve classifications for the individual stars are listed in column (5) of Table 3. Although there is only one star in this sample with a type $\mathrm{C}$ light curve, we decided to retain the class because the light curve is distinctive in appearance and because published light curves of classical Cepheids with periods near 10 days reveal other stars that would fit into this class.

In columns (6) and (7) we give the intensity means of the $V$ and $R$ magnitudes, while the amplitudes in $V$ and $R$ are tabulated in columns (8) and (9). We have also estimated the phase of minimum light and listed it in column (10).

In Figure 1 we present plots of the light curves. Most of the plots include all of the data. However, for the stars with no unique period (indicated by footnotes $\mathrm{c}$ and $\mathrm{h}$ in Table 3), we have plotted the only more recent data that fit the adopted period.

We have fitted Fourier series to the light curves. Again, when there was no unique period we have only used the recent data for which the period in Table 3 is valid. The order of the adopted fit for each star was selected by examining the scatter about the fitted curve and to avoid overfitting of the data. The Fourier parameters, defined in the usual way (Simon \& Lee 1981), are listed in Table 4. The errors of the parameters were calculated with the formulae given by Peterson (1986). The rather large uncertainties in the parameters for some of the stars are attributable to the intrinsic scatter in their light curves. Thus, a much greater amount of data would be needed to reduce them. In two cases, QQ Per and ET Vul, a third-order fit was required to avoid overfitting the data. Hence, no values are given for $R_{41}$ or $\phi_{41}$. Gaps in the light curve precluded a meaningful fit for AL Sct.

To measure the scatter in the light curves (listed in the last column of Table 4), we have used the standard deviations of 
the individual data points about a fitted curve. In most cases, these were calculated from the scatter of the Behlen Observatory data about a Fourier fit to the photometry from the literature. In cases where the form of the light curve differs between our data and the older data, we used the current data only. For AL Sct a reliable estimate of the scatter was possible by fitting to the populated intervals of phase.

\section{DISCUSSION}

\subsection{Light-Curve Morphology}

Our classification of light curves by appearance (col. [5] of Table 3) was done to assess the usefulness of photometry in distinguishing between type I and type II Cepheids. To gauge our success, Table 5 displays the frequencies of the various light-curve types for the GCVS classes and for the high- and low- $Z$ stars.

An examination of the upper part of Table 5 shows that most of the stars with type A or B light curves are classed as type II Cepheids in the GCVS (CWA, CWA:, or CW:), while the majority of the type $\mathrm{C}, \mathrm{D}$, and $\mathrm{Db}$ light curves correspond to stars classified as classical Cepheids (DCEP). If we restrict our attention to stars with periods longer than 12 days (lower part of Table 5), our classifications, based solely on lightcurve appearance, produce nearly perfect agreement with the GCVS. The only exception is PP Aql, which is listed as DCEP in the GCVS but has an A-type light curve. The significance of this agreement is unclear given that the classifications in the GCVS are based on various considerations, including lightcurve morphology.

A more objective comparison can be made by considering the high- $Z$ and low- $Z$ Cepheids that are distinguished according to a consistent, well-defined criterion. The majority of stars with A or B light curves are high- $Z$ Cepheids, while a majority of the $\mathrm{C}, \mathrm{D}$, and $\mathrm{Db}$ light curves correspond to low- $Z$ stars. However, there are a significant number of exceptions regardless of whether the sample is limited to periods longer than 12 days or not. Since we expect to find some type II Cepheids near the Galactic plane and since our sample was selected with a bias toward type II Cepheids, it is likely that some, or perhaps all, of the low- $Z$ stars with A and B light curves are, in fact, type II Cepheids. The high- $Z$ stars with type $\mathrm{D}$ or Db light curves are perhaps more surprising. Three of these, AP Her, $\mathrm{BH}$ Oph, and AL Vir, have periods less than 12 days, while the remainder, EV Aql, DR Cep, and EU Cyg, have longer periods. We will return to these stars below.

In Figure 2 we have plotted the Fourier parameters $R_{31}, R_{41}$, $\phi_{21}$, and $\phi_{31}$ against phase. There is no apparent separation between high- and low- $Z$ stars in the $R_{21}$ and $\phi_{41}$ diagrams, so they are not shown. In the case of $\phi_{41}$ this may be the consequence of the relatively large errors in that parameter. Since $\phi_{21}$ and $\phi_{31}$ are phases, we are free to arbitrarily add multiples of $2 \pi$ to them. Some authors have done so to preserve continuous sequences of points in the Fourier diagrams. However, when the sequences become separated by approximately $2 \pi$ they may appear to be distinct but, in fact, overlap. In addition, there is sometimes no objective way to determine whether to add $\pm 2 \pi$ to a phase parameter for an individual star. To avoid ambiguity and confusion, we have plotted $\phi_{21}$ and $\phi_{31}$ in the range from 0 to $2 \pi$. Thus, in looking at Figures $2 c$ and $2 d$ it is necessary to remember that they wrap over from the top to the bottom.

In Figure 2 high- $Z$ Cepheids are represented by filled symbols, while low- $Z$ Cepheids are plotted as open symbols. To prevent excessive crowding, we have only plotted error bars
TABLE 5

Light-CuRve Types

\begin{tabular}{|c|c|c|c|c|c|}
\hline Classification & $\mathrm{A}$ & B & $\mathrm{C}$ & $\mathrm{D}$ & $\mathrm{Db}$ \\
\hline \multicolumn{6}{|c|}{ All Stars } \\
\hline DCEP . & 2 & 0 & 1 & 2 & 8 \\
\hline $\mathrm{CWA}^{\mathrm{a}}$ & 8 & 6 & 0 & 4 & 0 \\
\hline 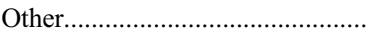 & 2 & 0 & 0 & 1 & 1 \\
\hline Low-Z & 6 & 2 & 1 & 3 & 7 \\
\hline High-Z & 6 & 4 & 0 & 4 & 2 \\
\hline
\end{tabular}

Stars with Periods Longer than 12 Days

\begin{tabular}{|c|c|c|c|c|c|}
\hline - & 1 & 0 & 0 & 2 & \\
\hline .............................. & 7 & 6 & 0 & 0 & 0 \\
\hline 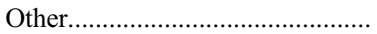 & 1 & 0 & 0 & 1 & 1 \\
\hline Low-Z & 4 & 2 & 0 & 2 & 5 \\
\hline 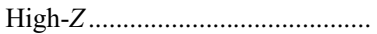 & 5 & 4 & 0 & 1 & 2 \\
\hline
\end{tabular}

a Includes CW: and CWA:

for selected points that are relevant in the discussion below. Points corresponding to a few individual stars are identified by the first part of their variable star names.

We have also plotted the Fourier parameters for the subset of stars selected by Simon \& Moffett (1985) from the tabulation of Moffett \& Barnes (1985) (triangles) and values of $\phi_{21}$ and $\phi_{31}$ for type II Cepheids listed by Fernie \& Ehlers (1999) (squares). These added data were included to provide additional guidance in interpreting the diagrams, but, with a single exception, we will not consider them in the discussion below.

Looking first at the amplitude ratios in Figures $2 a$ and $2 b$, we have drawn lines to separate most of the low- $Z$ from most of the high- $Z$ stars in the left-hand half of the period range. There are too few longer period stars to extend the dividing line beyond about 32 days. More than three-quarters of the low- $Z$ objects lie above the line and more than two-thirds of the high$Z$ objects lie below the line in both diagrams. This does not provide as clear-cut a separation of the two groups as we would have desired. However, it does suggest that the conclusion of Fernie \& Ehlers (1999) that "the amplitude ratios, $R_{i 1}$, were useless in this regard" was unduly pessimistic.

Of the five high- $Z$ Cepheids in the upper region of Figure $2 a$, three, EU Cyg, AP Her, and DR Cep, are above the line by a statistically significant amount. EU Cyg and DR Cep are also significantly above the dividing line in Figure $2 b$. On the other hand, none of the low- $Z$ Cepheids in either panel lie below the dividing line by a statistically significant amount.

In Figures $2 c$ and $2 d$ we have not drawn dividing lines because there are obvious sequences that are clearly separated. In Figure $2 c$ we note a sequence of mainly low- $Z$ stars extending nearly horizontally from a $P \approx 10$ days and $\phi_{21} \approx 4.4$ to $(25$ days, 5.0). A second sequence above the first [(14 days, 5.8) to (25 days, 5.4)] is composed mainly of high- $Z$ stars. However, the dozen points at the lower left of the plot represent the upper end of that sequence, which has wrapped over from the top. Both types of stars are present in that region. The two sequences may merge at longer periods, but there are too few stars to delineate the behavior beyond about 30 days. Similarly, in Figure $2 d$ two sequences can be distinguished [(12 days, 1.3) to (32 days, 3.5) and (12 days, 2.1) to (25 days, 4.2$)$ ] although they overlap at periods shorter than about 17 days. The behavior in both diagrams is similar to what was described earlier by Fernie \& Ehlers (1999) and Zakrewski et al. (2000). 

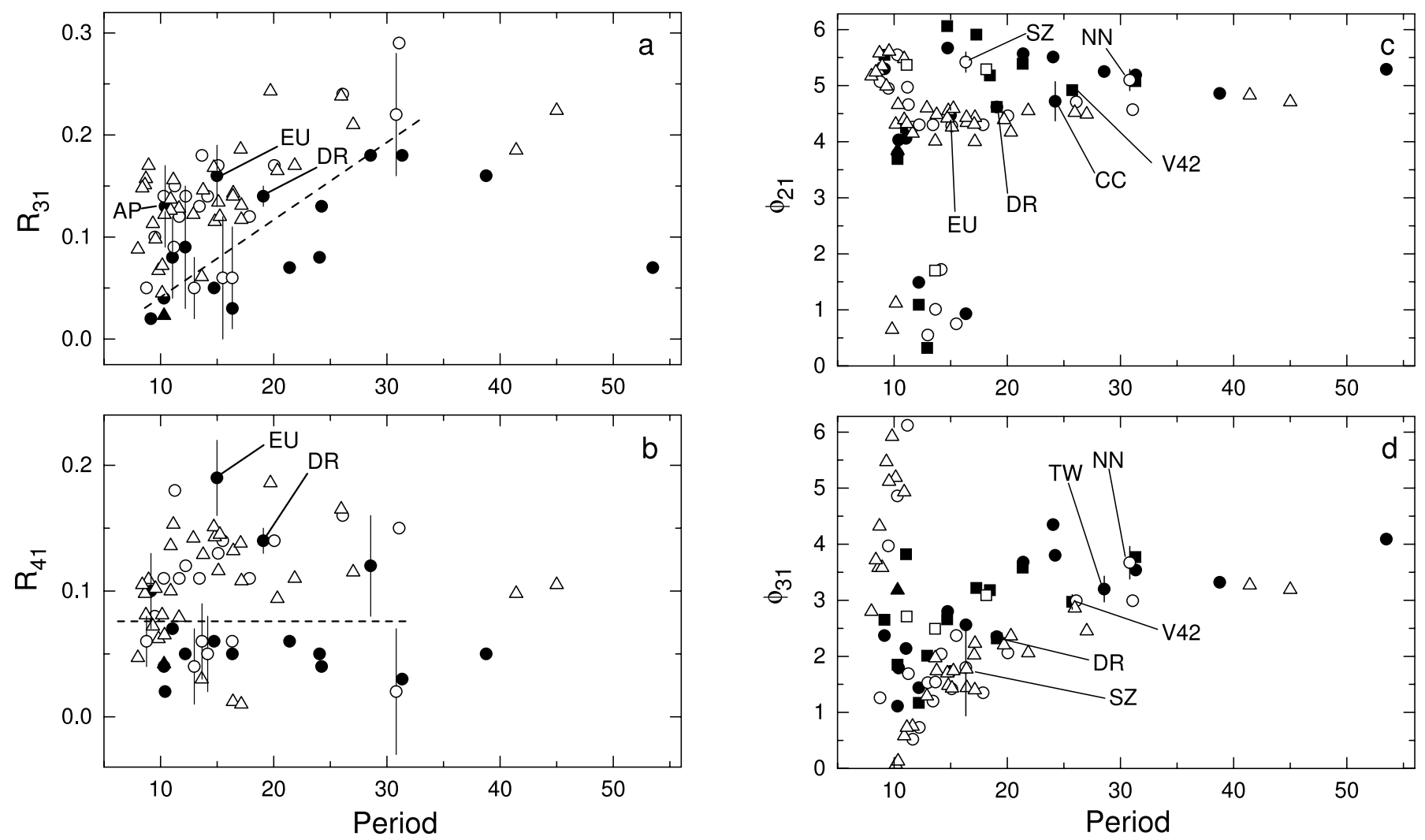

FIG. 2.-Four of the Fourier parameters plotted against period. The symbols indicate the sources of the data as follows: (circles) the present paper, (triangles) Moffett \& Barnes (1985), and (squares) Fernie \& Ehlers (1999). Filled symbols represent high- $Z$ stars, while open symbols represent low- $Z$ stars. Stars discussed in the text are identified by the first part of their variable star names.

The high- $Z$ stars EU Cyg, DR Cep, and CC Lyr are members of the lower sequence in Figure $2 c$ at a statistically significant level (note that the error bars are smaller than the symbols for the first two of these stars). In Figure $2 d$ DR Cep is in the lower sequence, but EU Cyg falls into the region where the sequences are not distinct. Star V42 in M5, which comes from Fernie \& Ehlers (1999), presents something of a puzzle. Although it is in the sequence of low- $Z$ stars in both Figures $2 c$ and $2 d$, it is a member of M5, which is clearly a Population II object. This may just indicate that the two sequences have already merged at a period of 25 days.

\subsection{Light-Curve Stability}

It has been known for many years that there is a tendency for type II Cepheids to exhibit more scatter in their light curves than is the case for classical Cepheids. To investigate this, two parameters are displayed in Figure 3. Again we have used different symbols for high- $Z$ and low- $Z$ objects.

In Figure $3 a$ we have plotted $|\Delta P|$, the difference between the periods from Table 3 and those listed in the GCVS, against period. The errors in these differences will be dominated by our period determinations, so the uncertainties listed in column (3) were used for the error bars. Since the periods from the GCVS are generally based on data that predates that used in deriving the periods in Table 3, this difference is a measure of the stability of the periods. Obviously, we cannot distinguish between short- and long-term changes. In addition, if the periods fluctuate over time (as opposed to a continuous lengthening or shortening) the values of $|\Delta P|$ plotted in Figure 3 will contain some spuriously small values.

We note that the high- $Z$ stars predominate among the higher values of $|\Delta P|$. There are seven high- $Z$ stars above the hori- zontal line at $|\Delta P|=0.015$ but only a single low- $Z$ star. On the other hand, half of the high- $Z$ stars fall in the lower region, and they constitute one-third of the stars there. As noted above, some small values of $|\Delta P|$ will occur by chance; but it is clear that this parameter is far from an unambiguous diagnostic of type. It is interesting to note that DR Cep and EU Cyg, which were in the low- $Z$ regions of the Fourier diagrams, are also among that group in this diagram.

The light-curve scatter from column (14) of Table 4 is plotted against period in the bottom panel of Figure 3. Again we have drawn a line to divide the diagram into two regions, this time at $\sigma_{V}=0.045$. This provides a better segregation between the high- and low- $Z$ stars than was the case in Figure $3 a$. Again we noted that DR Cep is in the lower region, while EU Cyg is now in the upper region by a small, probably insignificant, amount.

We conclude that the scatter in the light curve or phase jitter does provide some guidance in the classification of stars. However, it is clearly not unambiguous and is mainly useful in identifying stars of particular interest. It is likely, however, that the parameters shown in Figure 3 will prove useful in combination with other information.

\subsection{Stars with Large Period Changes}

There are six stars (identified by footnotes $\mathrm{c}$ and $\mathrm{h}$ in Table 3 ) for which we were unable to determine a period valid over the 20-30 yr covered by the data cited in Table 1 . For these stars we have fitted a template light curve to subsets of the photometry to derive phases of maximum (following Hertzsprung 1919). The errors were calculated using the scheme described by Fernie (1989). We also included the $V$ data for IX Cas from Harris \& Welch (1989), which was not included in the analysis above. For each star we selected subsets of the photometric 

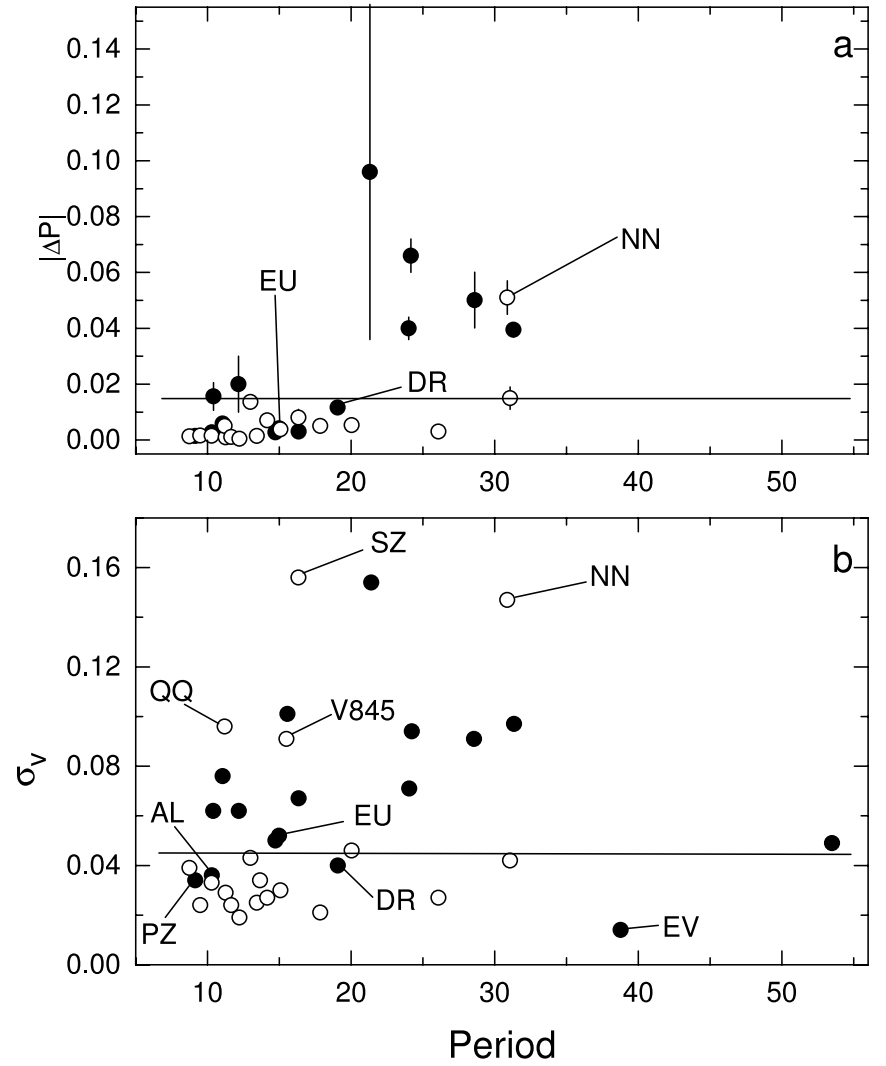

Fig. 3.- Stability parameters plotted against period. (a) Period difference, $|\Delta P|$; (b) light-curve scatter, $\sigma_{V}$. High- $Z$ stars are represented by filled circles, while low- $Z$ stars are plotted as open circles.

data spanning a reasonably short interval of time (less that 50 days in most cases) and containing at least three light-curve points. Although three data points may seem like a rather small number, if the photometry is of good quality and zero-point shifts are not significant, a well-determined date of maximum can be derived.

In Figure 4 we plot $O-C$, the difference between the fitted dates of maximum and dates calculated from a mean period, against the Julian Date. During intervals when a star has a constant period, the points in the $O-C$ diagram will lie along a straight line. It can be seen that none of the six stars shows evidence for a protracted interval of constant period.

Szabados $(1980,1981,1991)$ has studied the periods of AN Aur, IX Cas, and AP Her over intervals that mostly predate the data we have used. We did not include AN Aur in Figure 4 because we saw no sign of a significant period variation. This is consistent with Szabado's result. For the other two the behavior he shows is similar to what we have found. He remarked that the period changes he found in AP Her were the largest of the many Cepheids he had studied.

We note that orbital motion is a possible cause of period variations. However, the ranges in the $O-C$ values in Figure 4 are much too large to be explained by a companion of stellar mass.

We conclude that the period changes in these stars are intrinsic to the stars themselves. Five of the six stars are likely type II Cepheids that span the entire period range of our sample. There is nothing that obviously distinguishes them from the type II Cepheids with more stable periods. The sixth star, QQ Per, is a likely type I star, based on location and the

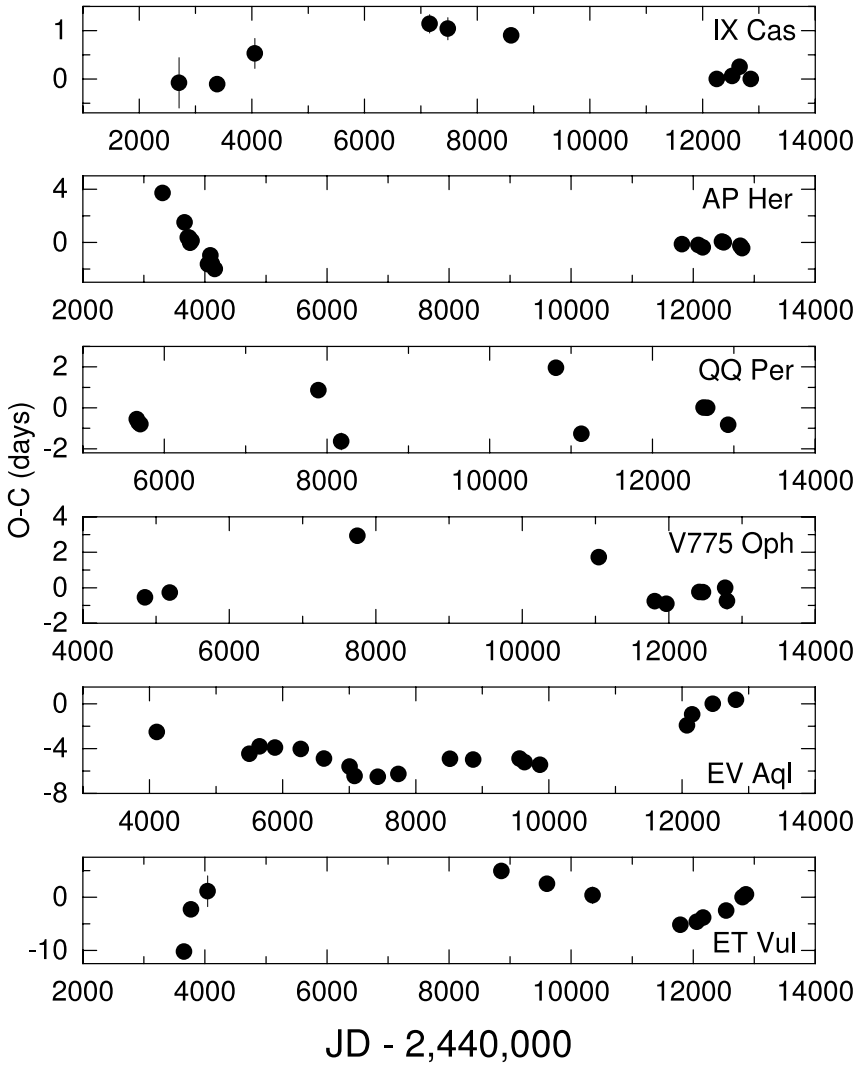

FIG. 4.-Differences between observed and calculated times of maximum plotted against Julian Date for six stars with exceptional period changes. Error bars are shown when they are larger than the plotted symbols. The calculated dates of maximum are based on mean periods over the whole range of epochs rather than the periods listed in Table 3 .

light-curve properties discussed above. Thus, we are unable to offer an explanation for this behavior on the part of a minority of type II Cepheids and one type I Cepheid.

\subsection{High-Z Stars with Type I Characteristics}

It is reasonable to assume that most of the high- $Z$ stars are type II Cepheids and most of the low- $Z$ stars are classical Cepheids. It then follows that the morphological types of the light curves and the locations of individual stars in Figures 3 and 4 can be used to discriminate between type I and type II Cepheids. These factors predominantly indicate that DR Cep and EU Cyg are type I Cepheids, generally at a level that is highly significant statistically. To these we can add IX Cas, AL Vir, AP Her, and CC Lyr, for which the predominance of the evidence indicates a type I classification, although with less certainty. All of these stars are high- $Z$ Cepheids.

As noted above, short-period Cepheids with type I characteristics have been identified well away from the Galactic plane. We suggest that DR Cep and EU Cyg and perhaps some of the other four stars mentioned in the previous paragraph are likely to be examples of the same situation among long-period variables. Clearly, further investigation of these stars is warranted.

We made extensive use of the McMaster Cepheid Photometry and Radial Velocity Data Archive in both the selection of stars for the program and in locating photometric data for use in 
this paper. We are appreciative of the efforts on the part of Douglas Welch in providing this resource. Dr. Roger Kirby, chair of Physics and Astronomy at the University of Nebraska, has provided continued support for the operation of Behlen
Observatory and encouragement of our research. These efforts have been crucial to the success of this research and are very much appreciated. This work was supported in part by NSF grant AST 00-97353.
Balog, Z., Vinko, J., \& Kaszas, G. 1997, AJ, 113, 1833

Berdnikov, L. N. 1986, Perem. Zvezdy, 22, 369 1987, Perem. Zvezdy, 22, 530

1992a, Astron. Astrophys. Trans., 2, 1

1992b, Astron. Astrophys. Trans., 2, 31

1992c, Astron. Astrophys. Trans., 2, 43

1992d, Astron. Astrophys. Trans., 2, 107

1992e, Astron. Astrophys. Trans., 2, 157

1992f, AZh Pis'ma, 18, 325

1993a, AZh Pis'ma, 19, 210

1993b, Perem. Zvezdy, 23, 64

Berdnikov, L. N., \& Turner, D. G. 1995, AZh Pis'ma, 21, 803

Berdnikov, L. N., \& Voziakova, O. V. 1995, AZh Pis'ma, 21, No. 5

Berdnikov, L. N., \& Yakubov, S. D. 1993, Perem. Zvezdy, 23, 47

Diethelm, R. 1990, A\&A, 239, 186

Fernie, J. D. 1989, PASP, 101, 225

Fernie, J. D., \& Ehlers, P. 1999, AJ, 117, 1563

Ferraz-Mello, S. 1981, AJ, 86, 619

Harris, H. C. 1980, Ph.D. thesis, Univ. Washington 1985a, in IAU Colloq. 82, Cepheids: Theory and Observations, ed.

B. F. Madore (Cambridge: Cambridge Univ. Press), 232 1985b, AJ, 90, 756

Harris, H. C., \& Wallerstein, G. 1984, AJ, 89, 379

Harris, H. C., \& Welch, D. L. 1989, AJ, 98, 981

Hertzsprung, E. 1919, Astron. Nachr. 210, 17

\section{REFERENCES}

Kholopov, P. N. 1985, General Catalogue of Variable Stars, Volumes 1 and 2 (4th ed.; Moscow: Nauka)

. 1987, General Catalogue of Variable Stars, Volume 3 (4th ed.; Moscow: Nauka)

Kovacs, G., \& Buchler, J. R. 1988, ApJ, 334, 971

Kwee, K. K. 1967, Bull. Astron. Inst. Netherlands, 19, 260

Moffett, T. J., \& Barnes, T. F. 1984, ApJS, 55, 389 1985, ApJS, 58, 843

Payne-Gaposchkin, C. 1956, Vistas in Astronomy, Volume 2, ed. A. Beer (Oxford: Pergamon), 1142

Pel, J. W. 1976, A\&AS, 24, 413

Peterson, O. J. 1986, A\&A, 170, 59

Schmidt, E. G. 1991, AJ, 102, 1766

Schmidt, E. G., Chab, J. R., \& Reiswig, D. E. 1995, AJ, 109, 1239

Schmidt, E. G., Langen, S., Lee, K. M., Johnston, D., Newman, P. R., \& Snedden, S. A. 2003a, AJ, 126, 2495

Schmidt, E. G., Lee, K. M., Johnston, D., Newman, P. R., \& Snedden, S. A. 2003b, AJ, 126, 906

Schmidt, E. G., \& Seth, A. 1996, AJ, 112, 2769

Simon, N. R., \& Lee, A. S. 1981, ApJ, 248, 291

Simon, N. R., \& Moffett, T. J. 1985, PASP, 97, 1078

Szabados, L. 1980, Commun. Konkoly Obs., 76, 1 -. 1981, Commun. Konkoly Obs., 77, 1

- 1991, Commun. Konkoly Obs., 96, 123

Zakrzewski, B., Ogloza, W., \& Moskalik, P. 2000, Acta Astron., 50, 387 